\title{
ANÁLISE PARA PEQUENOS SINAIS DE UM SISTEMA CA COMPOSTO DE INVERSORES CONECTADOS EM PARALELO
}

\author{
Ernane Antônio Alves Coelho* \\ ernane@ufu.br
}

\author{
Porfírio Cabaleiro Cortizo ${ }^{\dagger}$ \\ porfirio@cpdee.ufmg.br
}

\section{Pedro Francisco Donoso Garcia ${ }^{\dagger}$}
*Faculdade de Engenharia Elétrica, Universidade Federal de Uberlândia Campus Santa Mônica, 38400-902 Uberlândia, MG, BRASIL

${ }^{\dagger}$ Centro de Pesquisa e Desenvolvimento em Engenharia Elétrica, Universidade Federal de Minas Gerais, Campus Pampulha, 31270-901 Belo Horizonte, MG, BRASIL

\begin{abstract}
This paper presents the small signal analysis for parallel connnected inverters in stand-alone AC supply systems. The control technique of the inverters is based on frequency and voltage droops, which depends on the local variable measurements and do not need control interconnections. Simulation and experimental results show that system is well represented by the small signal model, which becomes stability studies easier.
\end{abstract}

KEYWORDS: Voltage inverters, parallel connection, small signal stability, frequency and voltage drop.

\section{RESUMO}

Este trabalho mostra a análise de estabilidade para pequenos sinais de um sistema CA composto de vários inversores de tensão conectados em paralelo sem a presença de uma barra infinita. O controle de cada unidade inversora é baseado nas curvas características frequência versus potência ativa e tensão versus potência reativa, o qual não depende da comunicação entre as respectivas unidades de controle. Os resultados de simulação

Artigo submetido em 05/12/00

1a. Revisão em 22/03/01

Aceito sob recomendação do Ed. Assoc. Prof. Edson H. Watanabe e experimentais comprovam a eficácia do modelo para pequenos sinais, facilitando os estudos de estabilidade do sistema.

\section{INTRODUÇÃO}

A estabilidade dinâmica de Sistemas Elétricos de Potência tem sido objeto de vários estudos nas últimas décadas. Undrill (1968) mostra como realizar a análise para pequenos sinais de um sistema composto por um número arbitrário de máquinas síncronas conectadas em paralelo, usando as técnicas da teoria moderna de controle, onde o sistema é descrito por um conjunto de equações diferenciais no espaço de estados na forma:

$$
[\dot{X}]=[A][X]
$$

Undrill (1968) descreve um método de construção da matriz $[A]$ de um sistema multimáquinas a partir das submatrizes que descrevem individualmente os elementos do sistema. Usando um método análogo, este trabalho descreve como contruir a matriz $[A]$ de um sistema composto por um número arbitrário de inversores conectados em paralelo. O controle de cada inversor é baseado nas curvas características $\omega$ versus $\mathrm{P}$ e $\mathrm{V}$ versus Q, o qual permite a distribuição de carga entre as várias unidades. Uma característica importante deste controle 
é que somente variáveis medidas localmente são usadas, dispensando a comunicação entre as respectivas unidades de controle de cada inversor, como pode ser visto em Tuladhar et al. (1997); Divan et al. (1991); Coelho et al. (1999); Coelho et al. (2000b) e Coelho et al. (2000a). Esta característica confere ao sistema um alto grau de confiabilidade.

\section{ESQUEMA DE CONTROLE}

O esquema de controle de cada unidade inversora conectada em paralelo pode ser visto na figura 1. A referência senoidal para os controladores mais internos ao sistema é obtida através de um bloco gerador, onde a frequência do sinal e a amplitude do mesmo são definidas a partir das curvas características $\omega$ versus $\mathrm{P}$ e $\mathrm{V}$ versus $\mathrm{Q}$, respectivamente. As potências ativa e reativa são obtidas no bloco de cálculo das potências, o qual utiliza um algoritmo computacional e um filtro passa-baixa.

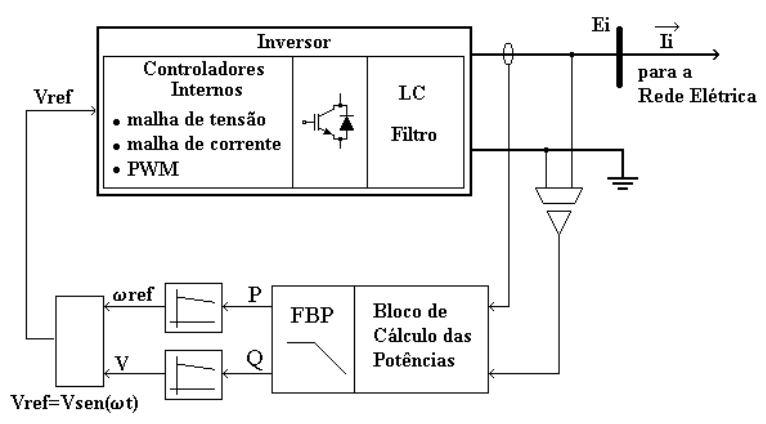

Figura 1: Unidade inversora conectada ao sistema

\section{ANÁLISE PARA PEQUENOS SINAIS DE CADA INVERSOR}

A frequência de saída $\omega$ e a tensão de saída $E$ do inversor são controladas através das curvas características definidas pelas equações 2 e 3 , respectivamente, as quais são apresentadas na figura 2 .

$$
\begin{aligned}
& \omega=\omega_{o}-k_{p} P \\
& E=E_{o}-k_{v} Q
\end{aligned}
$$

Para impor as leis de controle definidas pelas equações 2 e 3 , é preciso medir a potência ativa e reativa de saída do inversor. O respectivo bloco de medição utiliza um filtro passa-baixa, cuja banda é muito inferior a banda passante dos controladores internos do inversor(malha

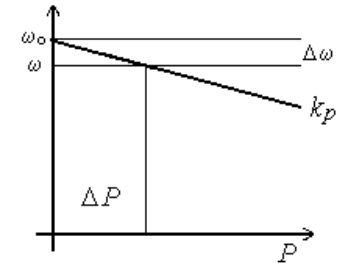

Potencia Ativa x Frequencia

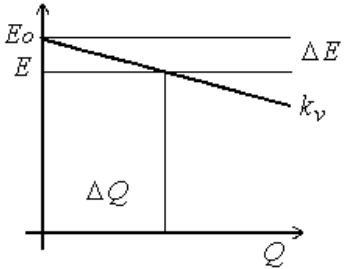

Potencia Reativa $x$ Tensão
Figura 2: Curvas características $\omega$ versus $\mathrm{P}$ e V versus $\mathrm{Q}$

de tensão e corrente) e praticamente determina o comportamento dinâmico da distribuição dos fluxos de potência. Além disso, a banda passante dos controladores internos pode ser melhorada através de várias técnicas, como pode ser visto em Ryan e Lorenz (1995). Portanto, para efeito deste estudo, o inversor pode ser considerado como uma fonte de tensão ideal, onde tem-se o controle de frequência e amplitude. Assim, as potências ativa e reativa obtidas no bloco de medição podem ser representadas através das equações 4 and 5 , onde $\omega_{f}$ é a frequência de corte do filtro de medição.

$$
\begin{aligned}
P_{\text {med }}(s) & =\frac{\omega_{f}}{s+\omega_{f}} P(s) \\
Q_{\text {med }}(s) & =\frac{\omega_{f}}{s+\omega_{f}} Q(s)
\end{aligned}
$$

Portanto, como poder ser visto em Coelho et al. (1999), linearizando-se as equações de controle 2 e 3 :

$$
\begin{aligned}
\Delta \omega(s) & =-\frac{k_{p} \omega_{f}}{s+\omega_{f}} \Delta P(s) \\
\Delta E(s) & =-\frac{k_{v} \omega_{f}}{s+\omega_{f}} \Delta Q(s)
\end{aligned}
$$

Assim, a partir das equações acima, tem-se

$$
\begin{aligned}
\Delta \dot{\omega} & =-\omega_{f} \Delta \omega-k_{p} \omega_{f} \Delta P \\
\Delta \dot{E} & =-\omega_{f} \Delta E-k_{v} \omega_{f} \Delta Q
\end{aligned}
$$

onde $\Delta$ representa um pequeno desvio na respectiva variável em relação ao ponto de equilíbrio.

Considerando um sistema comum de eixos d-q para todos os inversores, pode-se representar o vetor $\vec{E}$ como:

$$
\vec{E}=e_{d}+j e_{q}
$$


onde

$$
\begin{aligned}
e_{d} & =\operatorname{Ecos}(\delta) \\
e_{q} & =\operatorname{Esen}(\delta) \\
\delta & =\operatorname{arctg}\left(\frac{e_{q}}{e_{d}}\right)
\end{aligned}
$$

Linearizando a equação para $\delta$, o qual representa a posição angular do vector $\vec{E}$, tem-se

$$
\Delta \delta=\frac{\partial \delta}{\partial e_{d}} \Delta e_{d}+\frac{\partial \delta}{\partial e_{q}} \Delta e_{q}
$$

assim, segue que

$$
\Delta \delta=m_{d} \Delta e_{d}+m_{q} \Delta e_{q}
$$

onde

$$
\begin{aligned}
& m_{d}=-\frac{e_{q}}{e_{d}^{2}+e_{q}^{2}} \\
& m_{q}=\frac{e_{d}}{e_{d}^{2}+e_{q}^{2}}
\end{aligned}
$$

Desde que

$$
\Delta \omega(s)=s \Delta \delta(s)
$$

isto implica que

$$
\Delta \omega=m_{d} \Delta \dot{e_{d}}+m_{q} \Delta \dot{e_{q}}
$$

Considerando

$$
E=|\vec{E}|=\sqrt{e_{d}^{2}+e_{q}^{2}}
$$

pode-se linearizar a equação 20 , assim

$$
\Delta E=n_{d} \Delta e_{d}+n_{q} \Delta e_{q}
$$

onde

$$
\begin{aligned}
& n_{d}=\frac{e_{d}}{\sqrt{e_{d}^{2}+e_{q}^{2}}} \\
& n_{q}=\frac{e_{q}}{\sqrt{e_{d}^{2}+e_{q}^{2}}}
\end{aligned}
$$

Consequentemente

$$
\Delta \dot{E}=n_{d} \Delta \dot{e_{d}}+n_{q} \Delta \dot{e_{q}}
$$

Resolvendo o sistema de equações formado pelas expressões $9,19,21$, e 24 para as variáveis $\Delta \dot{e}_{d}$ e $\Delta \dot{e}_{q}$, tem-se

$$
\begin{gathered}
\Delta \dot{e_{d}}=\frac{n_{q}}{m_{d} n_{q}-m_{q} n_{d}} \Delta \omega+\frac{m_{q} n_{d} \omega_{f}}{m_{d} n_{q}-m_{q} n_{d}} \Delta e_{d} \\
+\quad \frac{m_{q} n_{q} \omega_{f}}{m_{d} n_{q}-m_{q} n_{d}} \Delta e_{q}+\frac{k_{v} m_{q} \omega_{f}}{m_{d} n_{q}-m_{q} n_{d}} \Delta Q \\
\Delta \dot{e_{q}}=\frac{n_{d}}{m_{q} n_{d}-m_{d} n_{q}} \Delta \omega+\frac{m_{d} n_{d} \omega_{f}}{m_{q} n_{d}-m_{d} n_{q}} \Delta e_{d} \\
+\quad \frac{m_{d} n_{q} \omega_{f}}{m_{q} n_{d}-m_{d} n_{q}} \Delta e_{q}+\frac{k_{v} m_{d} \omega_{f}}{m_{q} n_{d}-m_{d} n_{q}} \Delta Q
\end{gathered}
$$

Considerando as equações 8,25 , e 26, obtem-se a equação de estado 27, a qual descreve o comportamento de cada inversor.

$$
\left[\begin{array}{c}
\Delta \dot{\omega}_{i} \\
\Delta \dot{e}_{d i} \\
\Delta \dot{e}_{q i}
\end{array}\right]=\left[M_{i}\right]\left[\begin{array}{c}
\Delta \omega_{i} \\
\Delta e_{d i} \\
\Delta e_{q i}
\end{array}\right]+\left[C_{i}\right]\left[\begin{array}{c}
\Delta P_{i} \\
\Delta Q_{i}
\end{array}\right]
$$

\section{ANÁLISE PARA PEQUENOS SINAIS DO SISTEMA COMPLETO}

A fim de facilitar a compreensão desta análise, foi considerado um sistema composto por dois inversores conectados a uma rede, a qual é mostrada na figura 3 , mas tal análise pode ser estendida para um número arbitrário de inversores.

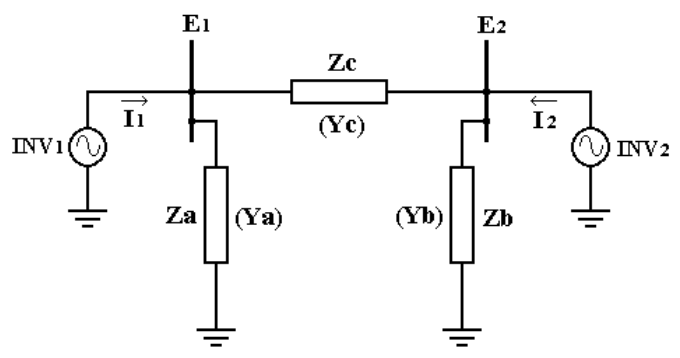

Figura 3: Sistema composto de 2 unidades inversoras

Desde que a variação de frequência na rede é considerada muito pequena, a rede pode ser completamente definida por uma equação matricial de nós utilizando as respectivas admitâncias entre os nós.

$$
\left[\begin{array}{c}
\overrightarrow{I_{1}} \\
\overrightarrow{I_{2}}
\end{array}\right]=\left[\begin{array}{cc}
Y_{a}+Y_{c} & -Y_{c} \\
-Y_{c} & Y_{b}+Y_{c}
\end{array}\right]\left[\begin{array}{c}
\overrightarrow{E_{1}} \\
\overrightarrow{E_{2}}
\end{array}\right]
$$

Transformando a equação complexa $28 \mathrm{em}$ sua forma real

$$
\left[\begin{array}{c}
i_{d 1} \\
i_{q 1} \\
i_{d 2} \\
i_{q 2}
\end{array}\right]=\left[\begin{array}{cccc}
G_{11} & -B_{11} & G_{12} & -B_{12} \\
B_{11} & G_{11} & B_{12} & G_{12} \\
G_{21} & -B_{21} & G_{22} & -B_{22} \\
B_{21} & G_{21} & B_{22} & G_{22}
\end{array}\right]\left[\begin{array}{c}
e_{d 1} \\
e_{q 1} \\
e_{d 2} \\
e_{q 2}
\end{array}\right]
$$


A equação acima pode ser escrita simbolicamente como

$$
[i]=\left[Y_{s}\right][e]
$$

Linearizando a expressão algébrica acima, tem-se

$$
[\Delta i]=\left[Y_{s}\right][\Delta e]
$$

Considerando as expressões para a potência ativa e reativa fornecida em cada inversor

$$
\begin{aligned}
P_{i} & =e_{d i} i_{d i}+e_{q i} i_{q i} \\
Q_{i} & =e_{d i} i_{q i}-e_{q i} i_{d i}
\end{aligned}
$$

Isto implica que

$$
\begin{array}{r}
{\left[\begin{array}{c}
\Delta P_{1} \\
\Delta Q_{1} \\
\Delta P_{2} \\
\Delta Q_{2}
\end{array}\right]=\left[\begin{array}{cccc}
i_{d 1} & i_{q 1} & 0 & 0 \\
-i_{q 1} & i_{d 1} & 0 & 0 \\
0 & 0 & i_{d 2} & i_{q 2} \\
0 & 0 & -i_{q 2} & i_{d 2}
\end{array}\right]\left[\begin{array}{c}
\Delta e_{d 1} \\
\Delta e_{q 1} \\
\Delta e_{d 2} \\
\Delta e_{q 2}
\end{array}\right]} \\
+\left[\begin{array}{cccc}
e_{d 1} & e_{q 1} & 0 & 0 \\
e_{q 1} & -e_{d 1} & 0 & 0 \\
0 & 0 & e_{d 2} & e_{q 2} \\
0 & 0 & e_{q 2} & -e_{d 2}
\end{array}\right]\left[\begin{array}{c}
\Delta i_{d 1} \\
\Delta i_{q 1} \\
\Delta i_{d 2} \\
\Delta i_{q 2}
\end{array}\right]
\end{array}
$$

a qual na sua forma simbólica é

$$
[\Delta S]=\left[I_{s}\right][\Delta e]+\left[E_{s}\right][\Delta i]
$$

Substituindo 31 em 35, tem-se

$$
[\Delta S]=\left(\left[I_{s}\right]+\left[E_{s}\right]\left[Y_{s}\right]\right)[\Delta e]
$$

A equação de estado para o sistema completo pode ser escrita como (ver equação 27)

$$
\begin{gathered}
{\left[\begin{array}{c}
\Delta \dot{\omega}_{1} \\
\Delta e_{d 1} \\
\Delta e_{q 1} \\
\Delta \dot{\omega}_{2} \\
\Delta e_{d 2} \\
\Delta e_{q 2}
\end{array}\right]=\left[\begin{array}{cc}
M_{1} & 0 \\
0 & M_{2}
\end{array}\right]\left[\begin{array}{c}
\Delta \omega_{1} \\
\Delta e_{d 1} \\
\Delta e_{q 1} \\
\Delta \omega_{2} \\
\Delta e_{d 2} \\
\Delta e_{q 2}
\end{array}\right]+} \\
{\left[\begin{array}{cc}
C_{1} & 0 \\
0 & C_{2}
\end{array}\right]\left[\begin{array}{c}
\Delta P_{1} \\
\Delta Q_{1} \\
\Delta P_{2} \\
\Delta Q_{2}
\end{array}\right]}
\end{gathered}
$$

a qual escrita simbolicamente é

$$
[\Delta \dot{X}]=\left[M_{s}\right][\Delta X]+\left[C_{s}\right][\Delta S]
$$

Combinando 38 e 36, tem-se

$$
[\Delta \dot{X}]=\left[M_{s}\right][\Delta X]+\left[C_{s}\right]\left(\left[I_{s}\right]+\left[E_{s}\right]\left[Y_{s}\right]\right)[\Delta e]
$$

Considerando que

$$
\left[\begin{array}{l}
\Delta e_{d 1} \\
\Delta e_{q 1} \\
\Delta e_{d 2} \\
\Delta e_{q 2}
\end{array}\right]=\left[\begin{array}{llllll}
0 & 1 & 0 & 0 & 0 & 0 \\
0 & 0 & 1 & 0 & 0 & 0 \\
0 & 0 & 0 & 0 & 1 & 0 \\
0 & 0 & 0 & 0 & 0 & 1
\end{array}\right]\left[\begin{array}{c}
\Delta \omega_{1} \\
\Delta e_{d 1} \\
\Delta e_{q 1} \\
\Delta \omega_{2} \\
\Delta e_{d 2} \\
\Delta e_{q 2}
\end{array}\right]
$$

ou simbolicamente

$$
[\Delta e]=\left[K_{s}\right][\Delta X]
$$

Substituindo a equação 41 em 39, tem-se

$$
[\Delta \dot{X}]=[A][\Delta X]
$$

onde

$$
[A]=\left[M_{s}\right]+\left[C_{s}\right]\left(\left[I_{s}\right]+\left[E_{s}\right]\left[Y_{s}\right]\right)\left[K_{s}\right]
$$

A equação homogênea 42 descreve o movimento livre do sistema em função de pequenas perturbações em torno do ponto de equilíbrio, isto é, o comportamento de $\Delta \omega_{1}$, $\Delta e_{d 1}, \Delta e_{q 1}, \Delta \omega_{2}, \Delta e_{d 2}$, e $\Delta e_{q 2}$ em torno do ponto de operação definido por $\omega_{1}, e_{d 1}, e_{q 1}, \omega_{2}, e_{d 2}$, e $e_{q 2}$, dada uma condição inicial ligeiramente fora do ponto de equilíbrio. O fluxo de carga deve ser determinado a fim de se estabelecer o ponto de equilíbrio.

\section{RESULTADOS DE SIMULAÇÃO}

Foram realizadas algumas simulações a fim de se verificar a representatividade do modelo para pequenos sinais proposto. Dois exemplos para diferentes características $\omega$ versus $\mathrm{P}$ e $\mathrm{V}$ versus $\mathrm{Q}$ são apresentados.

\subsection{Exemplo I}

Os resultados de simulação foram obtidos considerando o sistema mostrado na figura 3, cujos parâmetros são apresentados na tabela 1. O "offset"das curvas características $\omega$ versus $\mathrm{P}$ e $\mathrm{V}$ versus $\mathrm{Q}$ foram ajustados de forma que cada inversor forneça a potência especificada na tabela 1 na frequência de $377 \mathrm{rd} / \mathrm{s}$.

Considerando que na entrada em operação as potências ativa e reativa em cada inversor são nulas, e que os vetores $\vec{E}_{1}$ e $\vec{E}_{2}$ estão em fase, pode-se construir a matriz $[A]$ do sistema e obter os respectivos autovalores, os quais 
Tabela 1: Parâmetros do Sistema e Ponto de Equilíbrio

\begin{tabular}{|l|c|c|}
\hline Variável & Valor & Unidade \\
\hline \hline Linha de transmissão $\left(Z_{c}\right)$ & $0,5+j 3$ & $\Omega$ \\
Carga local - inversor $1\left(Z_{a}\right)$ & $13+j 6$ & $\Omega$ \\
Carga local - inversor $2\left(Z_{b}\right)$ & $25+j 13$ & $\Omega$ \\
Freq. de corte fitro de medição $\left(\omega_{f}\right)$ & 37,7 & $\mathrm{rd} / \mathrm{s}$ \\
Inclinação da curva $\omega$ versus $P\left(k_{p}\right)$ & 0,0005 & $\mathrm{rd} / \mathrm{s} / \mathrm{W}$ \\
Inclinação da curva V versus $Q\left(k_{v}\right)$ & 0,0005 & $\mathrm{~V} / \mathrm{VAR}$ \\
Potência aparente inversor $1\left(P_{1}+j Q 1\right)$ & $806+j 384$ & $\mathrm{VA}$ \\
Potência aparente inversor $2\left(P_{2}+j Q 2\right)$ & $750+j 375$ & $\mathrm{VA}$ \\
Tensão de saída inversor $1\left(\overrightarrow{E_{1}}\right)$ & $127+j 0$ & $\mathrm{~V}($ eficaz) \\
Tensão de saída inversor $2\left(\overrightarrow{E_{2}}\right)$ & $129,9+j 4,7$ & $\mathrm{~V}$ (eficaz) \\
Corrente de saída inversor $1\left(\overrightarrow{I_{1}}\right)$ & $6,4-j 3$ & $\mathrm{~A}($ eficaz) \\
Corrente de saída inversor $2\left(\overrightarrow{I_{2}}\right)$ & $5,9-j 2,7$ & $\mathrm{~A}($ eficaz) \\
Frequência no ponto de equlíbrio & 377 & rd/s \\
\hline
\end{tabular}

são:

$$
\begin{aligned}
& \lambda_{1}=0,0 \\
& \lambda_{2}=-6,5 \\
& \lambda_{3}=-31,2 \\
& \lambda_{4}=-37,7 \\
& \lambda_{5}=-37,8 \\
& \lambda_{6}=-39,4
\end{aligned}
$$

O sistema possui seis pólos reais no semi-plano esquerdo e consequentemente o mesmo é estável e apresenta uma resposta amortecida. A figura 4 mostra a variação da frequência de cada inversor obtida através da equação 42 e da simulação do sistema no Pspice. Observa-se que o sistema é bem representado pelo modelo para pequenos sinais. É importante observar que a equação 42 determina os desvios em relação ao ponto de equilíbrio e o comportamento das variáveis do sistema é determinado pela seguinte equação:

$$
[X]=[X]_{\text {pontoeq. }}+[\Delta X(0)] e^{[A] t}
$$

Os fluxos de potência ativa e reativa são mostrados na figura 5. Observa-se que o sinal de potência do sistema simulado ainda apresenta oscilações em $120 \mathrm{~Hz}$, as quais não foram completamente eliminadas pelo filtro de medição. O fluxo de potência reativa é mais rápido que o de potência ativa, mas ambos apresentam uma resposta amortecida.

\subsection{Exemplo II}

Usando o mesmo procedimento adotado para o Exemplo I, mas agora incrementando $k_{p}$ e $k_{v}$ em 10 vezes, podese construir uma nova matriz $[A]$ e obter os respectivos

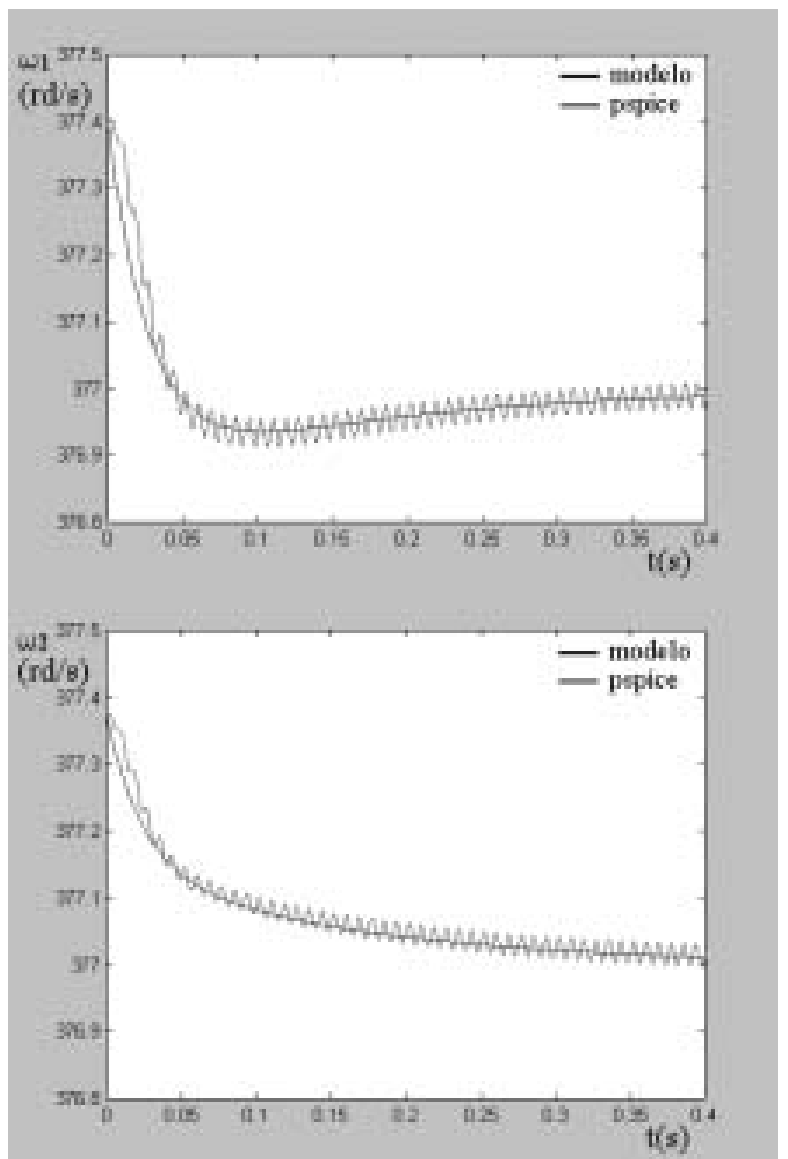

Figura 4: Variação na frequência dos inversores 1 e 2 Exemplo I

autovalores:

$$
\begin{aligned}
& \lambda_{1}=0,0 \\
& \lambda_{2}=-18,6+j 41,0 \\
& \lambda_{3}=-18,6-j 41,0 \\
& \lambda_{4}=-37,7 \\
& \lambda_{5}=-38,8 \\
& \lambda_{6}=-55,1
\end{aligned}
$$

Agora o sistema possui pólos com parte imaginária não nula, e portanto o mesmo apresenta uma resposta oscilatória. A parte real negativa dos respectivos pólos garante o amortecimento das oscilações. A figura 6 mostra a variação de frequência dos inversores definida pelo modelo para pequenos sinais e através de simulação no Pspice. Novamente, os resultados são muito próximos, atestando a eficência da análise para pequenos sinais.

As potências ativa e reativa são mostradas na figura 7 . Neste caso, a dinâmica de distribuição dos fluxos de po- 


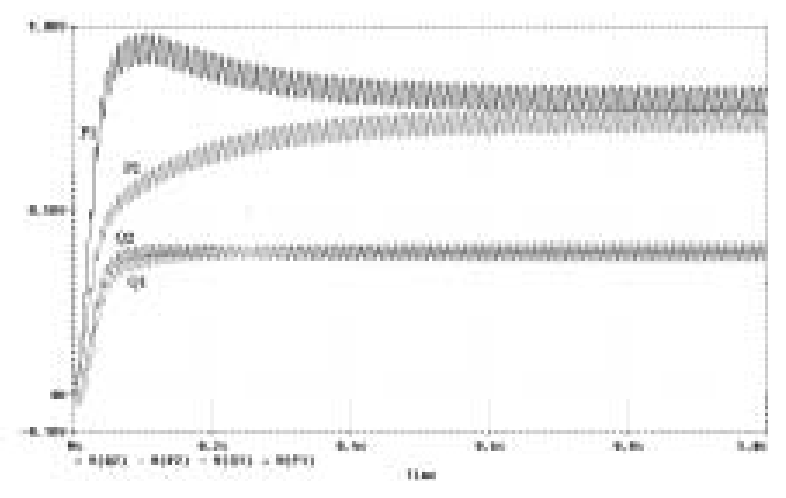

Figura 5: Potência ativa e reativa - Exemplo I

tência é mais rápida que no Exemplo I, mas apresenta oscilações.

Na determinação do ponto de equilíbrio, existe uma infinidade de conjuntos $e_{d 1}, e_{q 1}, e_{d 2}$ e $e_{q 2}$ que satisfazem a mesma condição de carga, isto é, o que importa é a posição relativa entre os vetores tensão $\vec{E}_{1}$ e $\vec{E}_{2}$ e não a posição absoluta dos mesmos. A mudança de $\vec{E}_{1}$ e $\vec{E}_{2}$ no eixo d-q (mantendo a posição relativa) altera a matriz $[A]$, mas não altera os autovalores. As variáveis de estado adotadas se justificam pela simplicidade resultante do modelo. Como pode ser visto em Chandorkar (1995), os ângulos $\Delta \delta_{i}$ formam um conjunto de variáveis não linearmente independentes, a matriz $[A]$ é singular e tem um autovalor nulo, o qual não tem interesse para estudos de estabilidade.

\section{RESULTADOS EXPERIMENTAIS}

A fim de validar os estudos teóricos, dois inversores PWM foram contruídos. O esquema de cada inversor é mostrado na figura 8. Este consiste de um inversor monofásico, com uma malha interna de corrente e uma malha externa de tensão utilizando compensadores PI. A parte sombreada consiste na malha de controle do fluxo de potência, a qual é implementada usando um computador PC e um sistema de aquisição de dados. Tem-se ainda uma malha de controle para eliminação da corrente média gerada devido a um pequeno "offset"presente na tensão de saída; e ainda um bloco PLL, usado para sincronizar o inversor com o sistema CA.

Primeiramente, o inversor 1 é conectado ao sistema CA e supre toda a carga. Então a chave SW1 é fechada, conectando o inversor 2 ao sistema, sendo que seus respectivos "gate drivers"se encontram desabilitados. A referência do inversor 2 é sincronizada com a tensão alternada que aparece no capacitor de filtro através do bloco PLL. Em

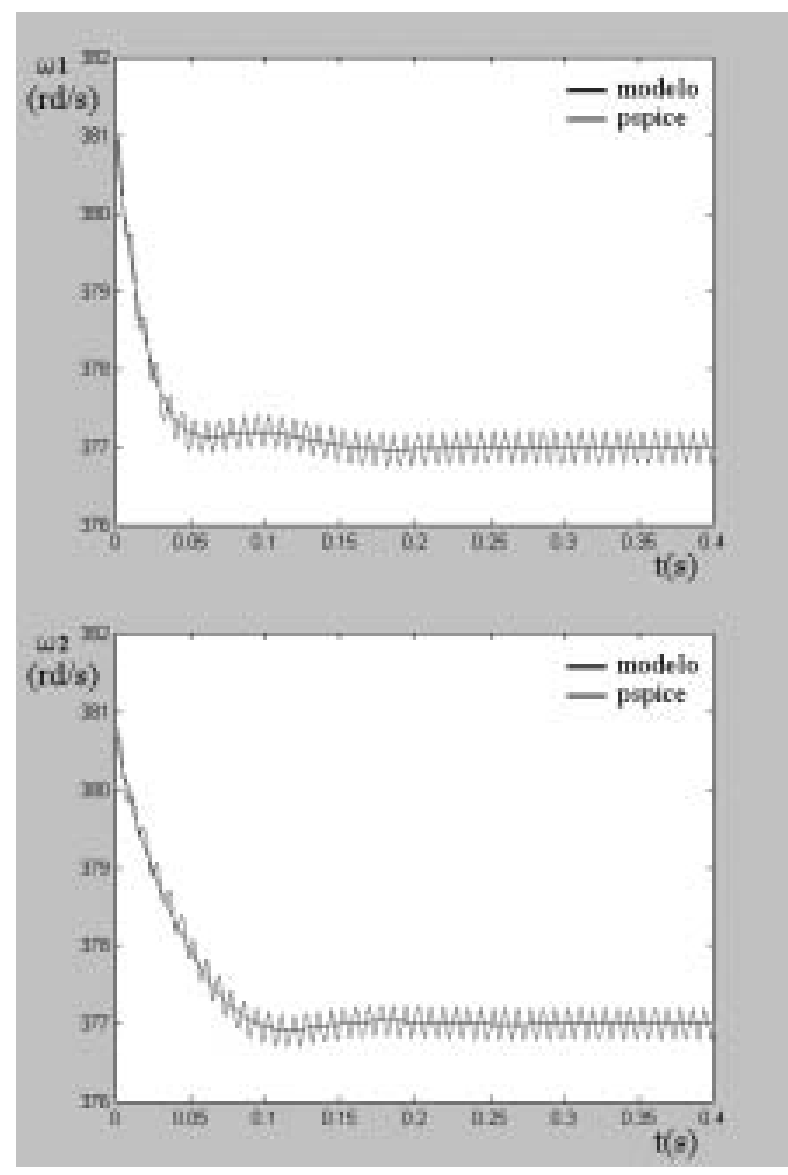

Figura 6: Variação na frequência dos inversores 1 e 2 Exemplo II

seguida, a chave SW2 ("software") é comutada da posição 1 para a posição 2 , sendo que os "gate drivers"do inversor 2 são simultaneamente habilitados através de uma porta paralela. Os resultados experimentais relativos a dois ensaios realizados para diferentes valores de $k_{p}$ e $k_{v}$ são apresentados a seguir.

\subsection{Ensaio I}

Os parâmetros usados neste ensaio são mostrados na tabela 2. A malha de controle do fluxo de potência e os "gate drivers"são habilitados 0,1 segundos depois que o sistema de aquisição começou a armazenar os dados.

A figura 9 mostra a forma de onda de freqüência obtida no ensaio experimental e através do modelo para pequenos sinais para o inversor 1 e 2 . Observamos uma excelente concordância entre os resultados experimentais e teóricos. Deve-se observar que a freqüência para o inversor 2 antes da habilitação do controle é definida pelo 


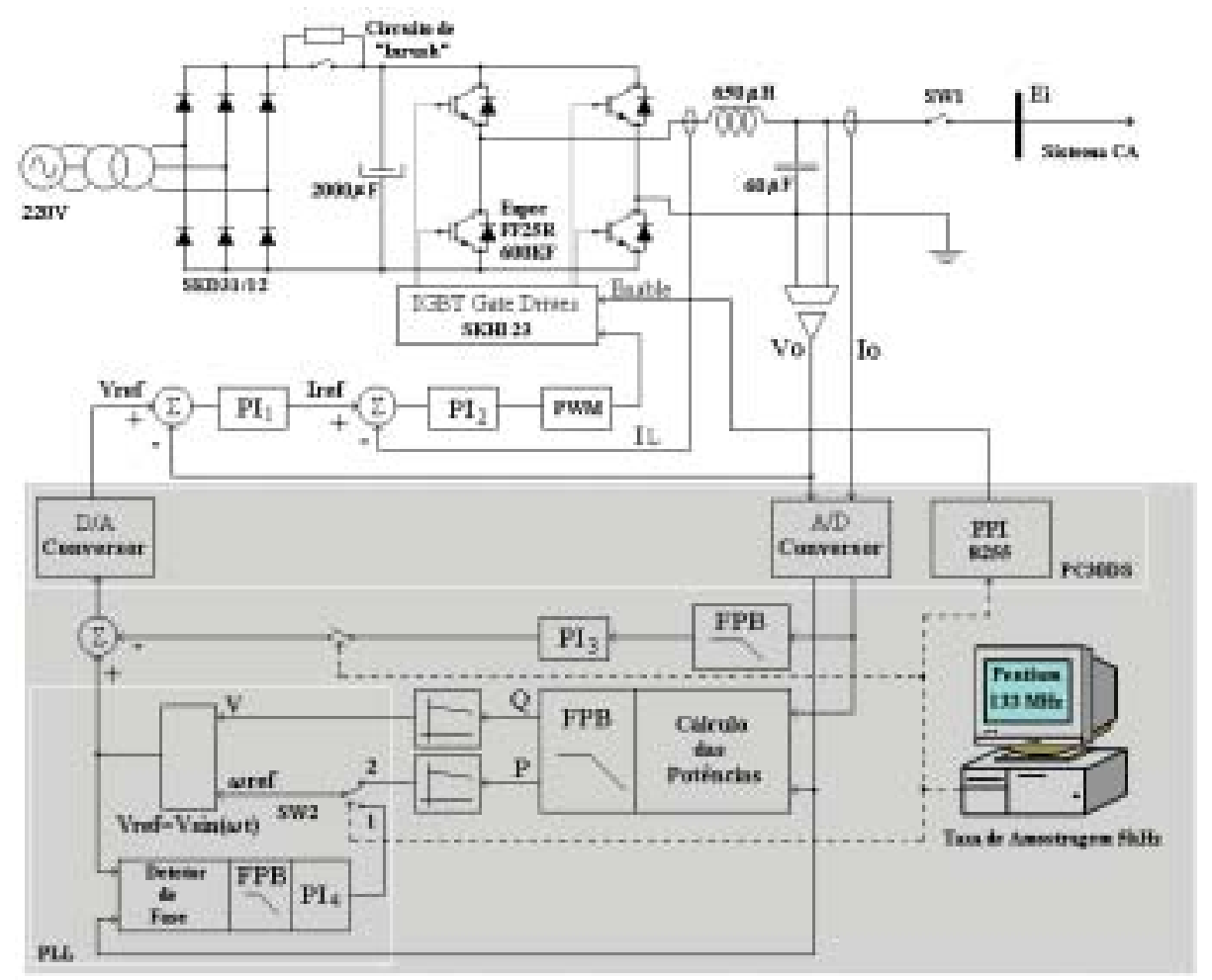

Figura 8: Esquema de cada inversor

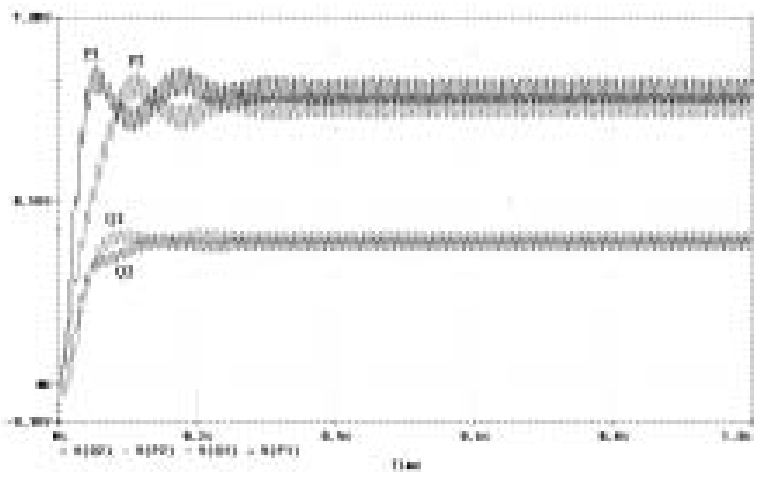

Figura 7: Potência ativa e reativa - Exemplo II
Tabela 2: Parâmetros do Sistema e Ponto de Equilíbrio

\begin{tabular}{|l|c|c|}
\hline Variável & Valor & Unidade \\
\hline \hline Linha de transmissão $\left(Z_{c}\right)$ & $0,2+j 3,1$ & $\Omega$ \\
Carga local - inversor $1\left(Z_{a}\right)$ & $25,7+j 27,2$ & $\Omega$ \\
Carga local - inversor $2\left(Z_{b}\right)$ & $52+j 9$ & $\Omega$ \\
Freq. de corte fitro de medição $\left(\omega_{f}\right)$ & 37,7 & $\mathrm{rd} / \mathrm{s}$ \\
Inclinação da curva $\omega$ versus $P\left(k_{p}\right)$ & 0,0005 & $\mathrm{rd} / \mathrm{s} / \mathrm{W}$ \\
Inclinação da curva V versus $Q\left(k_{v}\right)$ & 0,0005 & $\mathrm{~V} / \mathrm{VAR}$ \\
Potência aparente inversor $1\left(P_{1}+j Q_{1}\right)$ & $298+j 187$ & $\mathrm{VA}$ \\
Potência aparente inversor $2\left(P_{2}+j Q_{2}\right)$ & $280+j 180$ & $\mathrm{VA}$ \\
Tensão de saída inversor 1 $\left(\overrightarrow{E_{1}}\right)$ & $127+j 0$ & $\mathrm{~V}$ (eficaz) \\
Tensão de saída inversor 2 $\left(\overrightarrow{E_{2}}\right)$ & $130,3-j 1,2$ & $\mathrm{~V}$ (eficaz) \\
Corrente de saída inversor $1\left(\overrightarrow{I_{1}}\right)$ & $2,3-j 1,5$ & $\mathrm{~A}$ (eficaz) \\
Corrente de saída inversor $2\left(\overrightarrow{I_{2}}\right)$ & $2,1-j 1,4$ & $\mathrm{~A}$ (eficaz) \\
Freqüência no ponto de equilíbrio & 377 & rd/s \\
\hline
\end{tabular}

Na definição das condições iniciais a ser aplicada ao modelo, deve-se observar que o capacitor de filtro do inversor 2 atua como carga antes do mesmo ser ativado, o qual fornece reativo para o sistema.

A figura 11 mostra a distribuição dos fluxos de potência ativa e reativa para os inversores 1 e 2. Observa-se que inicialmente o inversor 1 supre toda a potência ativa, 


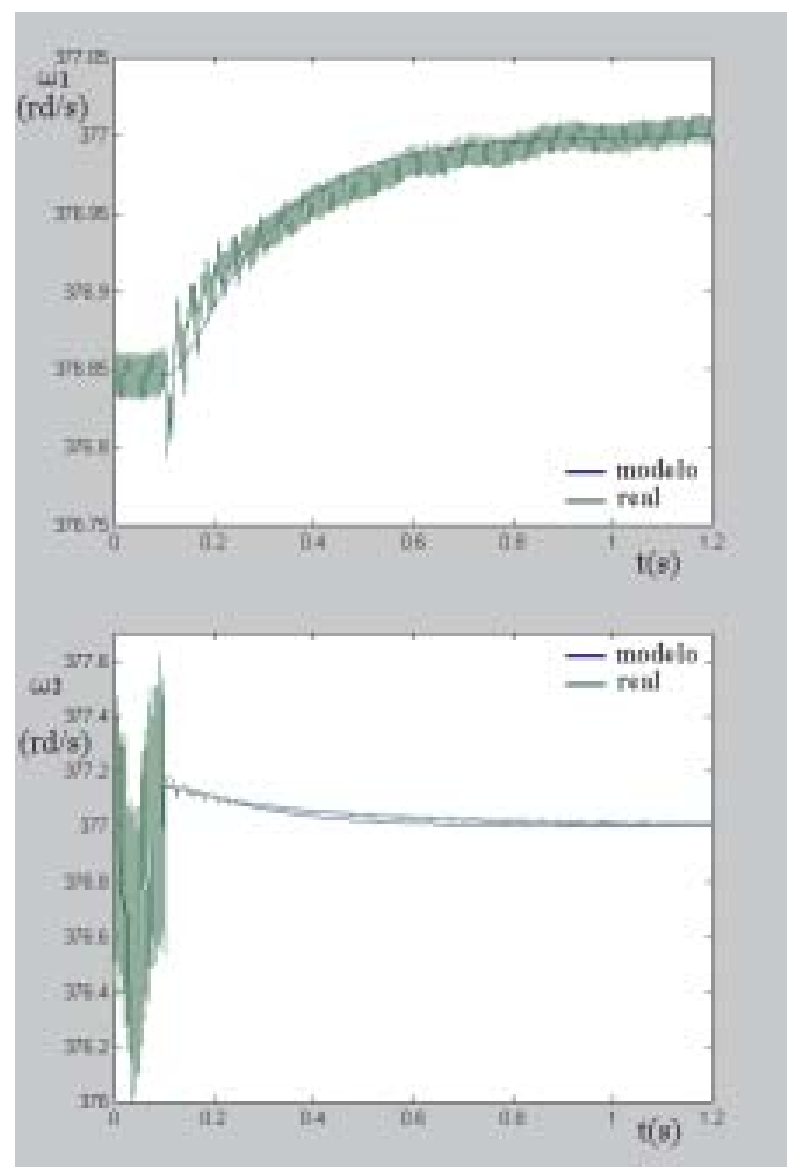

Figura 9: Freqüência real e do modelo

a qual é convenientemente distribuída com o inversor 2 após a conexão deste no sistema. Antes da entrada do inversor 2, o capacitor de filtro deste fornece praticamente todo o reativo requisitado pelo sistema. $\mathrm{O}$ valor de regime para as potências reativas apresentam alguma discordância em relação ao especificado na tabela 2 , mas é importante notar que um erro de 1,0 V implica num desvio de 2000 VAR, considerando uma inclinação da curva $\mathrm{V}$ versus $\mathrm{Q}$ de $0,0005 \mathrm{~V} / \mathrm{VAR}$.

A figura 12 apresenta as formas de onda de corrente e tensão para os inversores 1 e 2 nos instantes iniciais do ensaio. A escala de corrente está ampliada em 10 vezes. Observando a amplitude e fase da corrente em relação a tensão pode-se verificar a correspondência com os fluxos de ativo e reativo obtidos.

\subsection{Ensaio II}

Neste caso, os parâmetros usados no ensaio são os mesmos da tabela 2, exceto os coeficientes $k_{p}$ e $k_{v}$, os quais

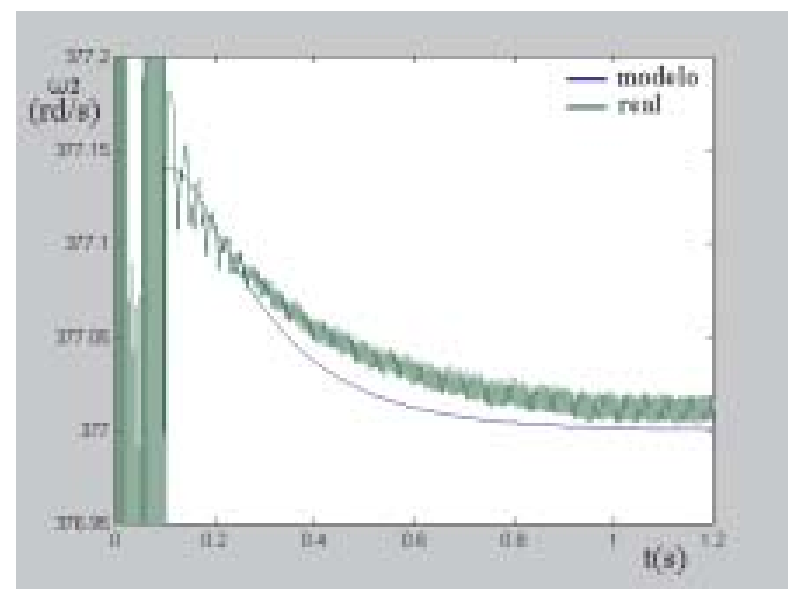

Figura 10: Freqüência real e do modelo para o inversor 2 detalhe da figura 9
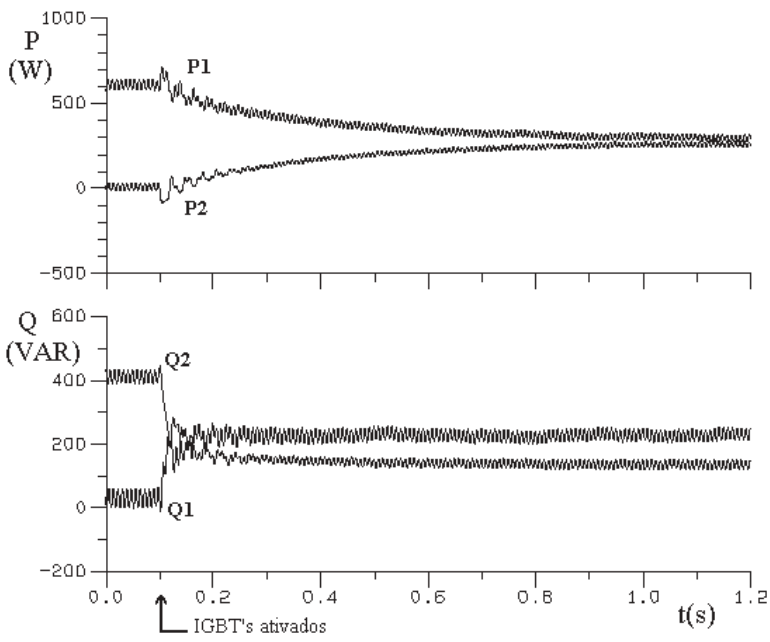

Figura 11: Potência ativa e reativa

foram incrementados em 10 vezes, ou seja, $k_{p}=0,005$ $\mathrm{rd} / \mathrm{s} / \mathrm{W}$ e $k_{v}=0,005 \mathrm{~V} / \mathrm{VAR}$. A malha de controle do fluxo de potência e os "gate drivers"são habilitados 0,05 segundos depois que o sistema de aquisição começou a armazenar os dados.

A figura 13 mostra a forma de onda de freqüência obtida no ensaio experimental e através do modelo para pequenos sinais para o inversor 1 e 2 . Novamente tem-se uma excelente concordância entre os resultados experimentais e teóricos. 


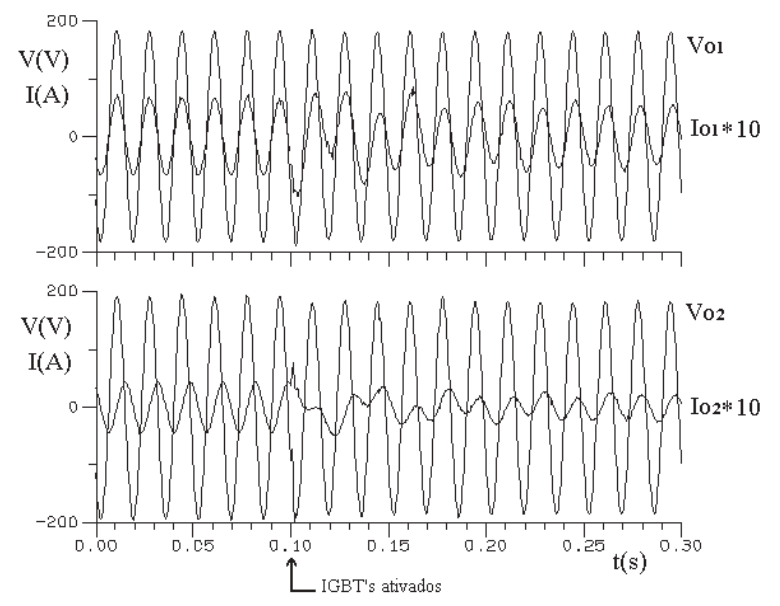

Figura 12: Corrente e tensão de saída em cada inversor

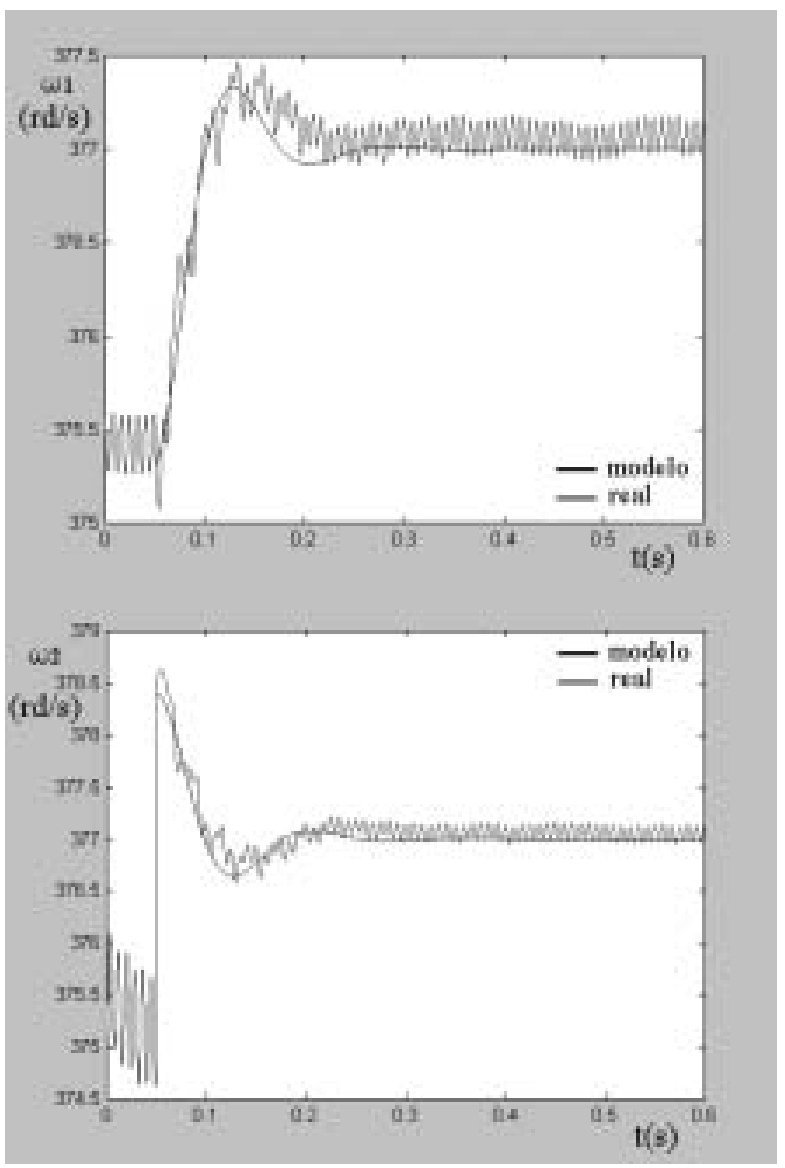

Figura 13: Freqüência real e do modelo

A figura 14 mostra a distribuição dos fluxos de potência ativa e reativa para os inversores 1 e 2 . Observa-se que a distribuição dos fluxos de potência se processa de maneira mais rápida que no ensaio I, mas apresenta um comportamento subamortecido.

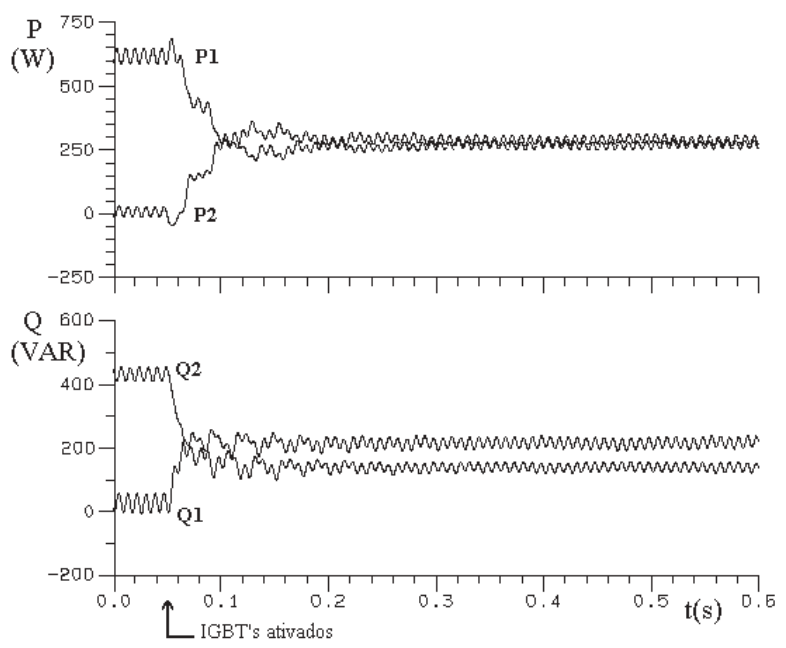

Figura 14: Potência ativa e reativa

As formas de onda de corrente e tensão para os inversores 1 e 2 são mostradas na figura 15 . A escala de corrente está ampliada em 10 vezes. Como no ensaio I, pode-se notar a correspondência com os fluxos de ativo e reativo obtidos.

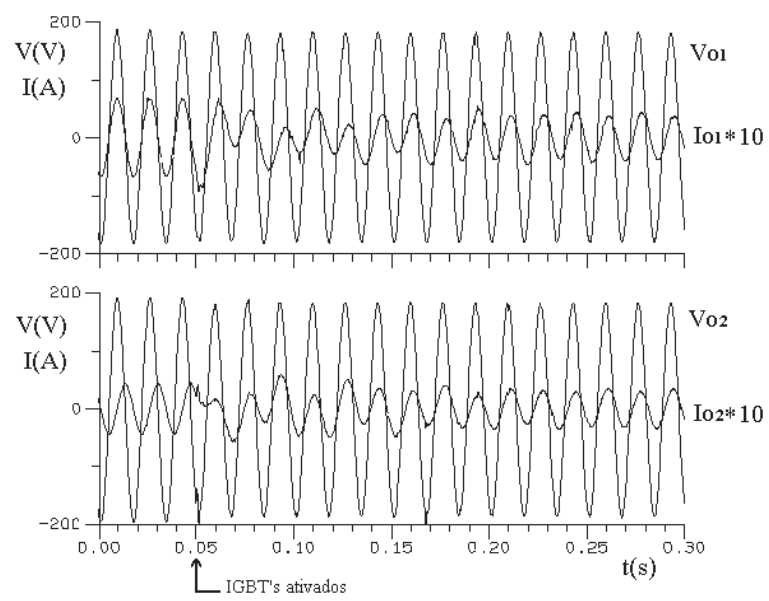

Figura 15: Corrente e tensão de saída em cada inversor

\section{CONCLUSÃO}

A análise para pequenos sinais de um sistema de inversores conectados a uma rede elétrica, sem a presença de 
uma barra infinita é apresentada, a qual facilita os estudos de estabilidade e desempenho. Os resultados de simulação e experimentais mostram que o sistema é satisfatoriamente representado pelo modelo para pequenos sinais obtido.

\section{REFERÊNCIAS}

Chandorkar, M. C. (1995). Distributed Uninterruptible Power Supply Systems, PhD thesis, University of Wiscosin-Madson.

Coelho, E. A. A., Cortizo, P. C. e Garcia, P. F. D. (1999). Small signal stability for single phase inverter connected to stiff ac system, IAS 99, Vol. 1, IEEE-IAS. CD-ROM.

Coelho, E. A. A., Cortizo, P. C. e Garcia, P. F. D. (2000a). Análise para pequenos sinais de um sistema ca composto de inversores conectados em paralelo, $C B A^{\prime}$ '2000, Vol. CDROM.

Coelho, E. A. A., Cortizo, P. C. e Garcia, P. F. D. (2000b). Small signal stability for parallel connected inverters in stand-alone ac supply systems, IAS'2000, Vol. CDROM.

Divan, D. M., Chandorkar, M. C. e Adapa, R. (1991). Control of parallel connected inverter in standalone ac supply systems, $I A S^{\prime} 91$, pp. 1003-1009.

Ryan, M. J. e Lorenz, R. D. (1995). A high performance sine wave inverter controller with capacitor current feedback and "back-emf"decoupling, Conf. Rec. IEEE-PESC, pp. 507-513. Atlanta, GA.

Tuladhar, A., Jin, H., Unger, T. e Mauch, K. (1997). Parallel operation of single phase inverter modules with no control interconnections, APEC'97, Vol. 1, pp. 94-100. Atlanta, GA.

Undrill, J. M. (1968). Dynamic stability calculations for an arbitrary number of interconnected synchronous machines, IEEE Transactions On Power Apparatus and Systems 87(3): 835-844. 